\title{
AVALIAÇÃO DO CICLO DE VIDA DE EDIFICAÇÕES ATRAVÉS DA UTILIZAÇÃO DA MODELAGEM DE INFORMAÇÕES DA CONSTRUÇÃO (BIM): ESTADO DA ARTE
}

\author{
Gabriel Conceição $^{(1)}$, Emerson Ferreira ${ }^{(1)}$, João Luiz Calmon ${ }^{(2)}$ \\ (1) Universidade Federal da Bahia, Salvador \\ (2) Universidade Federal do Espírito Santo, Espírito Santo
}

\begin{abstract}
Resumo
Embora ainda pouco disseminada, a integração entre a Avaliação do Ciclo de Vida (ACV) e do Custo do Ciclo de Vida (CCV) de edificações e a Modelagem de Informações da Construção (BIM) vem sendo uma área de tendência de pesquisas no setor de gestão das construções. Porém, para que a interoperabilidade entre essas áreas ocorra adequadamente, é necessária uma adoção do ACV / CCV integrado ao BIM ainda na fase inicial do projeto, momento em que a maioria das decisões de sustentabilidade deve ser tomada. O objetivo do presente trabalho é desenvolver uma revisão das pesquisas voltadas para a integração entre a avaliação do ciclo de vida e BIM, por meio de uma análise bibliométrica realizada baseada em bancos de dados como Scopus e Web of Science. Concluiu-se que a integração do BIM com ACV / CCV permite: a redução de recursos, esforços e a minimização da ocorrência de erros para a otimização do desempenho ambiental da edificação, mas ainda existem algumas limitações dessa integração com relação à interoperabilidade entre as ferramentas utilizadas e essa é uma questão fundamental para impulsionar ou usar o BIM integrado ao ACV e ao CCV. Portanto, o potencial do BIM no desenvolvimento de um projeto sustentável vai depender não apenas das tecnologias aplicadas, como da integração entre elas.
\end{abstract}

\section{Introdução}

A indústria da construção civil figura com destaque entre aquelas que apresentam um impacto substancial sobre o meio ambiente [1] e por seu alto consumo de energia e recursos naturais que resulta em impactos diretos e indiretos durante toda a vida útil do edifício [2].

O setor responde por cerca de $40 \%$ das emissões globais de $\mathrm{CO}_{2}$ e $40 \%$ do consumo de recursos naturais [3]. A fase operacional dos edifícios convencionais representa aproximadamente $80 \mathrm{a}$ 90\% do consumo de energia em todo seu ciclo de vida [4]. Por essa razão, essa fase merece a devida atenção dos gestores, particularmente na fase inicial do projeto, momento em que mudanças exigem menos energia e influenciam fortemente na sustentabilidade, na energia e no 
custo do ciclo de vida de um edifício [5]. Portanto, é essencial a aplicação de novas estratégias, como sistemas para aumentar a eficiência energética e reduzir os custos dos edifícios [2]. Um método que pode ser utilizado é a Avaliação do Ciclo de Vida (ACV) [2]. A ACV permite avaliar os impactos ambientais associados à construção, manutenção e demolição de edifícios e é cada vez mais aplicado para avaliar edifícios ao longo de seu ciclo de vida [6].

No âmbito de análise econômica há o Custo do Ciclo de Vida (CCV) que se refere a uma técnica de análise que abrange todos os custos associados a um produto desde a sua concepção à sua disposição, visando minimizar o custo de obter um certo nível de produção [7]. O principal incentivo para a aplicação de uma análise CCV é aumentar a possibilidade de redução de custos para a fase operacional, mesmo que seja necessário um aumento adicional no investimento inicial [8], obtendo-se assim um projeto viavelmente econômico para toda a vida útil do empreendimento.

Por outro lado, a inovação da tecnologia de modelagem de informações da construção (BIM) fornece um novo meio de prever, gerenciar e monitorar os impactos ambientais da construção e desenvolvimento do projeto por meio da tecnologia virtual de prototipagem / visualização em toda a vida útil de uma edificação [3]. O BIM possibilita apoiar decisões complexas, oferecendo oportunidades de vincular inúmeros aspectos e grandes quantidades de informações em um ambiente de dados comum [3].

Nos próximos anos a pressão nos mercados imobiliários por construções cada vez mais sustentáveis aumentará continuamente a demanda por indicadores internacionais e ferramentas de dados BIM de benchmarking para projeto, operação, manutenção e medição pós-ocupação eficientes [9]. Assim, a utilização da ACV e/ou CCV integrados ao BIM, pode permitir extrair quantitativos para estabelecer o inventário do ciclo de vida e possibilita auxílio na tomada de decisões complexas acerca dos impactos ambientais e custos gerados durante todo o ciclo de vida do edifício [10].

Diante do exposto acima, o presente trabalho busca desenvolver uma revisão das pesquisas voltadas para a integração entre a avaliação do ciclo de vida e de modelagem de informações da construção (BIM). Objetiva-se identificar as principais vantagens e desvantagens da integração do ACV e CCV com o BIM, limitações associadas a este uso e identificar as lacunas de literatura para direcionamento de trabalhos futuros.

\section{Metodologia}

A seleção do portfólio bibliográfico que embasou a revisão de literatura foi feita com uma busca nas bases de dados do Portal Capes - Scopus e Web of Science, por meio da combinação das palavras chaves: "Life cycle costing" ou "life cycle analysis" ou "Life cycle costing assessment" e "BIM" ou "building information modeling". A busca foi realizada pelas ferramentas das próprias bases de dados, usando como filtros: artigos com as palavras-chave nos títulos, palavras-chave ou resumos.

Foram encontrados 67 trabalhos na base Scopus, entre 2004 e 2019, e outros 8 na Web of Science, entre 2003 e 2019, relacionados ao tema de pesquisa. Gerando um banco de artigos bruto com 75 artigos na língua inglesa publicados em periódicos indexados com fator de impacto.

A primeira filtragem realizada foi a partir da leitura dos títulos, selecionando aqueles que estavam alinhados com a proposta deste trabalho, na segunda filtragem verificou-se os resumos 
dos trabalhos e a disponibilidade deles; assim o número de arquivos foi reduzido para 38 trabalhos de relevância internacional. Pode-se visualizar o processo empregado para a seleção de periódicos na Figura 1.

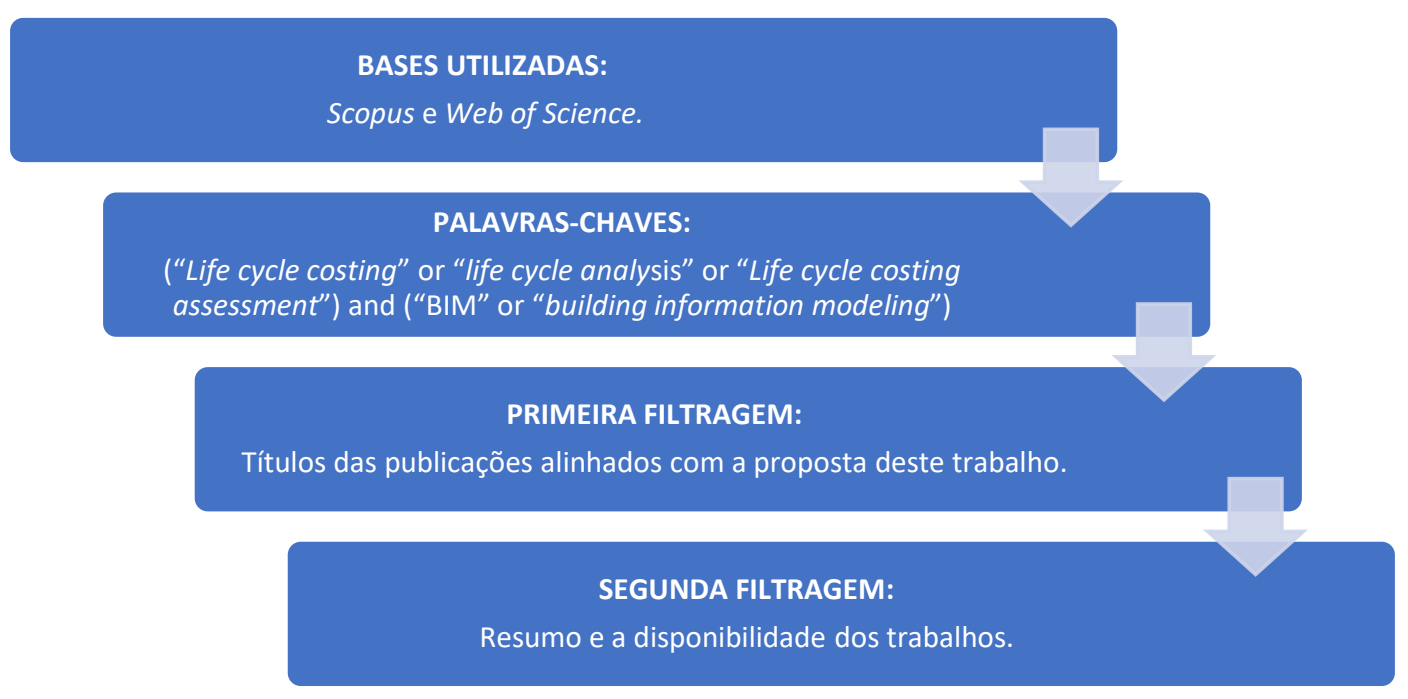

Figura 1: Metodologia de seleção dos periódicos.

\section{Resultados e Discussões}

Após a seleção e leitura dos artigos selecionados foi possível realizar uma análise sistemática sobre o que já foi estudado acerca da interoperabilidade entre a avaliação do ciclo de vida e da modelagem de informações da construção (BIM), assim foi possível identificar as principais vantagens e desvantagens da integração do ACV e CCV com o BIM, além das limitações associadas a este uso e posteriormente identificar as lacunas de literatura.

\subsection{Análise Bibliométrica}

Através do processo de busca foi possível realizar uma análise bibliométrica a respeito das pesquisas desenvolvidas na área, obtendo-se informações acerca das quantidades de publicações anuais, países, periódicos das publicações, principais autores e instituições.

O intervalo de publicação dos 38 trabalhos encontrados foi entre 2008 e 2019, sendo que os anos de 2009, 2012 e 2013 não houve publicações. Nos três primeiros anos houve apenas uma publicação anual, em 2014 e 2015 o número de trabalhos aumentou para dois, em 2016 foram três publicações e a partir de então o número de artigos publicados foi mais expressivo: seis em 2017, nove publicações em 2008 e treze em 2019.

Em relação aos países das publicações, Egito, Estados Unidos e Reino Unido foram os países com o maior número de publicações, três por país. A Bélgica, a Polônia, a República Checa e o Sudão são os seguintes com duas publicações de cada. Há trabalhos realizados em parcerias entre países, dentre eles vale destacar a Bélgica e Portugal, com duas publicações e o Brasil que publicou com a Austrália e o Canadá. Os demais países que aparecem na lista apresentaram apenas uma publicação.

Os periódicos com maior número de publicações foram a Sustainability, com oito publicações e o IOP Publishing, com sete trabalhos. Automation in Construction e Building and 
Environment também aparecem na lista com duas publicações cada. As demais publicações foram feitas por 19 periódicos distintos.

Com relação aos autores, foram identificados 102 pesquisadores na área, a maioria deles com apenas uma publicação. Entre os que possuíam mais publicações estão: Alexander Hollberg (Universidade de Hasselt, Bélgica), António Aguiar Costa (Universidade de Lisboa, Portugal), José D. Silvestre (Universidade de Lisboa, Portugal), Lincy Pyl (Universidade Livre de Bruxelas, Bélgica), Linda Hildebrand (Universidade Técnica de Aachen, Alemanha), Piotr Gradzinski (Universidade de Tecnologia da Pomerânia Ocidental, Polônia), Rúben Santos (Universidade de Lisboa, Portugal) e Walid Tizani (Universidade de Nottingham, Reino Unido), todos eles com duas publicações.

\subsection{Avaliação da integração entre BIM e ACV/CCV}

O uso da metodologia BIM possibilita a otimização de projetos, pois é nesse momento que mudanças são mais fáceis de serem implementadas. Desse modo, [11] concluíram que o desenvolvimento de análises detalhadas de aspectos ambiental, térmico, vento, sombra, visual bulk, ciclo de vida integradas com avaliações de impactos ambientais e de custos gerados pela construção de qualquer projeto por meio da Plataforma BIM faz com que considerações sustentáveis sejam pensadas e tomadas cada vez mais cedo no processo de projeto de empreendimentos.

É preciso mais esforço para preparar o sistema BIM para a realização de um retrofit em um edifício, mas vale a pena porque existem várias vantagens [12]. O modelo 3D, composto por elementos de superfície, permite o uso dos mesmos elementos de superfície para estimar o consumo e economia de energia e os custos iniciais de um possível retrofit, por exemplo.

A manutenção contínua do banco de dados BIM permite construir um sistema de informação que possa funcionar durante o ciclo de vida de um edifício. Esse sistema de informação, conectado à plataforma da Internet, permite que gestores tomem decisões confiáveis, possibilitando redução nos custos operacionais e de manutenção das instalações. Os cálculos e planos da adaptação podem ser documentados facilmente com a ajuda do sistema BIM [12].

Um estudo que gerou um modelo sustentável desenvolvido para avaliação de impactos ambientais incorporados a um projeto integrando-o ao uso de uma ferramenta de autoria BIM foi desenvolvido por Lee et al (2015). Os autores criaram uma grande biblioteca de materiais de construção sustentáveis e foi construída uma tabela de visão geral incorporada aos resultados da avaliação de impacto ambiental, para permitir que o usuário possa verificar os impactos ambientais em tempo real ao projetar um edifício usando a biblioteca de elementos de construção. A aplicabilidade do modelo foi verificada pela pequena taxa média de erros (5\%) entre os resultados da avaliação de impacto ambiental baseada neste modelo e aos modelos tradicionais em 2D [13].

Os benefícios de uma avaliação do ciclo de vida baseada em BIM incluem que informações como geometria de construção, estrutura, material, instalação e uso funcional são armazenadas no modelo BIM. Isso reduz tempo e custo para análise do desempenho ambiental do edifício. O benefício de adaptar a estrutura de tomada de decisão em um processo baseado em modelo BIM é a transparência das decisões de projeto com relação aos objetivos no início do projeto. No entanto, ele não define explicitamente as interdependências semânticas entre os parâmetros de projeto. As modificações necessárias do projeto ainda precisam ser testadas por profissionais especialistas na área ambiental [14]. 
Existem três abordagens principais para a integração do BIM com CCV e ACV. A primeira recorre a vários softwares para ambas as análises, a segunda exporta uma lista de dados de um modelo BIM e usa-a com bancos de dados externos e a terceira abordagem inclui informações de avaliações ambientais e econômica nos próprios modelos BIM [15].

Baseada na técnica de modelagem de recursos para determinar a sustentabilidade do projeto do edifício com estrutura de aço, Oti e Tizani discutiram uma estrutura integrada. Para os autores, o BIM apresenta oportunidades para integrar a modelagem de desempenho ambiental nos estágios iniciais do projeto de construção. A pesquisa concluiu que o processo utilizado e técnicas de modelagem de dados podem ser empregadas para modelar informações relacionadas à sustentabilidade para informar as decisões desde os estágios iniciais do projeto estrutural [16]. Foram desenvolvidos estudos onde foi explorado o potencial do BIM-ACV para avaliar o impacto ambiental de um edifício [17]. Os autores identificaram as soluções mais sustentáveis como materiais e orientação do edifício a partir do software Ecotect. Já Basbagill et al (2013) precisaram integrar sete ferramentas (DProfiller, eQUEST, SimaPro, Athena EcoCalculator, CostLab, Excel e ModelCenter) para desenvolver um estudo de ACV [18].

Outras pesquisas definiram os dados necessários para realizar uma análise de ACV e CCV e, em seguida, desenvolveram uma estrutura que integra essas metodologias com ferramentas BIM. No final, modelos BIM foram usados para exportar informações dos materiais como tipo, volume e peso que servem para alimentar uma planilha que conduz as análises de ACV e CCV [19].

Foi utilizado também um método integrado com o BIM, ACV e módulo de certificação e custo para obter o impacto ambiental de um edifício. O modelo BIM foi usado para gerar e exportar quantitativos que posteriormente foram conectados com um banco de dados externo desenvolvido pelos autores contendo informações ambientais por meio da ferramenta Athena Impact. A estrutura da certificação LEED foi empregada para obter dados de custo e potencial dos componentes de construção em aspectos de energia e projeto ambiental [20].

$\mathrm{Na}$ pesquisa desenvolvida por [21], quantitativos foram extraídos do modelo BIM para uma planilha que apresentava dados de CCV para diferentes tipos de elementos e materiais. Uma das observações resultantes foi que seria interessante integrar os dados de CCV aos elementos do modelo BIM em vez de usar bancos de dados externos.

A literatura existente se concentrou fortemente nos estágios de projeto e construção. É necessário integrar os manuais de manutenção e operação da instalação ao BIM para se obter um sistema sustentável [3]. A integração do BIM e do ACV pode ser um grande trunfo durante o processo de projeto / construção, ou seja, além da modelagem, componentes do sistema predial, zonas de acesso podem ser incluídas no modelo para garantir que o pessoal de operações e manutenção tenha acesso adequado a válvulas, painéis e equipamentos para manutenção [22].

Um trabalho mostrou que é possível realizar uma integração de ACV no BIM ao usar dados comuns entre a estrutura de dados para a realização da ACV e elementos BIM [10]. Para os autores, aplicando essa abordagem permite um cálculo integrado de BIM dos impactos incorporados aos materiais de construção nas fases iniciais do projeto. Usando uma variedade de opções possíveis de construção, é possível uma análise abrangente da construção como um todo e uma contribuição dos elementos construtivos para o impacto total, bem como a identificação de pontos de acesso específicos do projeto e potencial de melhoria de elementos de construção. 
Foi desenvolvido por Cavalliere et al (2018) um documento que identifica e codifica os parâmetros relevantes para executar a ACV de edifícios, que pode ser implementada no ambiente BIM, permitindo estruturar as informações de forma codificada e de maneira não redundante. Tornando possível, desse modo, executar ACV com base nos dados disponíveis no BIM, permitindo-se extrair informações diretamente do modelo de forma consistente, reduzindo o risco de erros, aproximações e omissões devido a dados inconsistentes ou ausentes [24].

A Tabela 1 traz resumidamente os principais benefícios observados nas pesquisas desenvolvidas na área relacionadas a integração do BIM com ACV e CCV.

Tabela 1: Benefícios da integração do BIM com ACV e CCV

\begin{tabular}{|c|c|c|}
\hline $\begin{array}{l}\text { Benefícios } \\
\text { Observados }\end{array}$ & Descrição & Artigos \\
\hline $\begin{array}{l}\text { Otimização de } \\
\text { projetos }\end{array}$ & $\begin{array}{l}\text { Possibilidade de extrair informações diretamente } \\
\text { do modelo, reduzindo o risco de erros, } \\
\text { aproximações e omissões. }\end{array}$ & $\begin{array}{l}{[11],[14],[16]} \\
\quad \text { e [24] }\end{array}$ \\
\hline $\begin{array}{l}\text { Auxílio para tomada } \\
\text { de dedisões }\end{array}$ & $\begin{array}{l}\text { Considerações sustentáveis são pensadas e } \\
\text { tomadas cada vez mais cedo no processo de projeto } \\
\text { de empreendimentos. }\end{array}$ & $\begin{array}{l}{[11],[14],[16]} \\
\text { e [22] }\end{array}$ \\
\hline $\begin{array}{l}\text { Estimativa de } \\
\text { quantidade, custo, } \\
\text { desempenho e } \\
\text { economia de materiais } \\
\text { e componentes. }\end{array}$ & $\begin{array}{l}\text { Por meio de informações como geometria de } \\
\text { construção, estrutura, material, instalação e uso } \\
\text { funcional no modelo BIM. }\end{array}$ & $\begin{array}{l}\text { [12], [17], [18], } \\
\text { [19], [20] e [21] }\end{array}$ \\
\hline
\end{tabular}

Compartilhamento de Inclusão no modelo BIM de informações que [10], [11], [12], informações por todo contribuam com pessoal de operações e [19] e [22] ciclo de vida da manutenção.

edificação

\begin{tabular}{|c|c|}
\hline $\begin{array}{l}\text { Avaliação de } \\
\text { impactos ambientais } \\
\text { em diferentes fases da } \\
\text { edificação }\end{array}$ & $\begin{array}{l}\text { Possibilidade do usuário verificar os impactos }[10],[13],[17] \text {, } \\
\text { ambientais em tempo real ao projetar um edifício, } \\
\text { considerando não apenas a fase de construção, mas } \\
\text { toda a vida útil do empreendimento. }\end{array}$ \\
\hline $\begin{array}{l}\text { Redução do tempo e } \\
\text { custo para análise do } \\
\text { desempenho } \\
\text { energético do edifício }\end{array}$ & $\begin{array}{l}\text { Inclusão de informações como geometria de [13], [14], [17], } \\
\text { construção, estrutura, material, instalação e uso [18], [19] e [21] } \\
\text { funcional no modelo BIM. }\end{array}$ \\
\hline $\begin{array}{l}\text { Elaboração de } \\
\text { biblioteca de } \\
\text { materiais e } \\
\text { componentes } \\
\text { sustentáveis }\end{array}$ & $\begin{array}{l}\text { Geração e exportação de informações e [13], [14], [17], } \\
\text { quantitativos dos materiais como: tipo, volume e [18], [19] e [21] } \\
\text { peso que servem para conduzir as análises de ACV } \\
\text { e CCV. }\end{array}$ \\
\hline
\end{tabular}


Conforme apresentado na Tabela 1, a integração entre o BIM e as análises de ACV e CCV possibilitam uma série de benefícios. É possível destacar como principais vantagens a otimização de projetos, o auxílio para tomada de decisões cada vez mais cedo no processo de projeto, a possibilidade de elaboração de biblioteca de materiais e componentes sustentáveis que permitem a geração de estimativa de quantidade, desempenho, economia e custo desses materiais e componentes, o compartilhamento de informações por todo ciclo de vida da edificação, a avaliação de impactos ambientais em diferentes fases da edificação e a redução do tempo e custo para análise do desempenho ambiental do edifício.

\subsection{Lacunas e pesquisas futuras}

O uso de modelos conceituais de BIM requer mais pesquisa sobre as informações necessárias para realizar a simulação de energia, bem como uma avaliação ambiantal do materiais de construção [10]. A integração da simulação de energia pode ser obtida por meio de ferramentas especializadas de simulação, bem como pelo uso de plug-ins de simulação de energia para interfaces de script. O desenvolvimento futuro deve incluir mais materiais BIM no Modelo $\mathrm{BIM}$, mais processos e diferentes cenários de ACV para estágios de fim de vida [23].

É necessário o desenvolvimento de bancos de dados abrangentes de elementos de construção com base em critérios comuns de desempenho funcional. Além disso, provisão de um banco de dados comum sobre elementos de construção poderia melhorar significativamente a avaliação nos estágios iniciais do projeto. O método é limitado à comparação das opções de construção disponíveis no banco de dados inicial da ACV. ACV expandida, abrangente e com bases de dados atualizadas são necessárias para apoiar a ampla aplicação do fluxo de trabalho de projeto e precisa incluir novas tecnologias e alternativas de materiais de construção [10].

Embora o estudo de [2] tenha como objetivo produzir edifícios com eficiência energética examinando a fase do ciclo de vida operacional dos edifícios, os autores apontam que a estrutura proposta pode ser facilmente expandida para cobrir todas as etapas de um ciclo de vida do edifício. Uma recomendação para trabalhos futuros seria considerar toda a vida útil do edifício, a fim de apontar confiabilidade nos resultados. Outra recomendação poderia ser investigar uma ampla variedade de componentes de construção que compõem o edifício, levando em consideração dados climáticos e fontes geográficas para cobrir mais regiões do mundo.

A análise não deve levar em consideração apenas a quantidade de materiais, mas também seu tipo (marca). Como os produtos apresentam diferentes fabricantes estes podem ter impactos ambientais e econômicos muito distintos. Nesse sentido, o BIM deve ser usado para promover a integração da cadeia de suprimentos e os fabricantes podem desempenhar um papel central no desenvolvimento de objetos digitais que contêm informações úteis para os projetos [15].

A Tabela 2 traz algumas das lacunas identificadas nas pesquisas relacionadas ao uso do BIM em conjunto do CCV e ACV. 
Tabela 2: Lacunas identificadas da integração do BIM com ACV e CCV

\begin{tabular}{|c|c|c|}
\hline Lacunas Identificadas & Descrição & Artigos \\
\hline $\begin{array}{l}\text { Desenvolvimento de } \\
\text { bancos de dados } \\
\text { abrangentes de } \\
\text { elementos de } \\
\text { construção } \\
\text { sustentáveis. }\end{array}$ & $\begin{array}{l}\text { Melhoria significativa da avaliação, consideração } \\
\text { não apenas da quantidade de materiais, mas também } \\
\text { seu tipo (marca), dos seus distintos impactos } \\
\text { ambientais e econômicos e levando em } \\
\text { consideração dados climáticos e fontes geográficas } \\
\text { para cobrir diferentes regiões do mundo. }\end{array}$ & $\begin{array}{l}{[10],[23],} \\
{[2] \text { e }[15]}\end{array}$ \\
\hline $\begin{array}{l}\text { Desenvolvimento de } \\
\text { plug-ins de simulação } \\
\text { de energia para } \\
\text { interfaces de script no } \\
\text { Modelo BIM }\end{array}$ & $\begin{array}{c}\text { Melhorias em relação à intregração do BIM e do } \\
\text { ACV/CCV e interoperabilidade entre as } \\
\text { ferramentas utilizadas }\end{array}$ & [10] e [15] \\
\hline $\begin{array}{l}\text { Aumento de pesquisas } \\
\text { que considerem toda a } \\
\text { vida útil do edifício até } \\
\text { o seu fim de vida. }\end{array}$ & $\begin{array}{l}\text { Muitos estudos focam nas fases de construção e na } \\
\text { fase operacional do ciclo de vida dos edifícios }\end{array}$ & [2] e [23] \\
\hline
\end{tabular}

Além das lacunas citadas na Tabela 2, outros desafios identificados na literatura, são: problemas de interoperabilidade entre ferramentas BIM e ferramentas de sustentabilidade; falta de estudos ontológicos, maior investigação teórica, nos campos da construção sustentável; falta de padrões e incentivos públicos para adoção do BIM dentro de uma indústria de construção sustentável e falta de bibliotecas BIM com objetos ricos em semântica.

Superar algumas dessas limitações e promover o uso do BIM como um repositório de informações que pode ser usado para gerenciamento de informações de projetos sustentáveis integrado com ACV / CCV[15].

\section{Conclusão}

A utilização de modelos BIM vem sendo crescente nos últimos anos, pois possibilita um aumento na qualidade do desempenho do projeto. A integração do BIM com a Avaliação do Ciclo de Vida apresenta como principais benefícios: a redução de recursos, esforços, além da minimização da ocorrência de erros para a otimização do desempenho ambiental da edificação. $\mathrm{O}$ uso de modelos virtuais integrados ao CCV permite avaliar se o investimento proporciona benefícios econômicos ao longo de sua vida útil. Além disso, também pode ser adotado para avaliar o retorno do investimento da adoção de medidas de conservação de energia, calculando as economias de energia operacional decorrentes do investimento inicial.

Existem algumas limitações dessa integração, destacam-se como desvantagens: questão da interoperabilidade e da análise integrada dos softwares, a transferência de informações de uma ferramenta de criação BIM para um programa de simulação energética é um processo unidirecional, qualquer alteração necessária no modelo de simulação de energia deve ser realizada no software de projeto em BIM. 
A interoperabilidade entre as ferramentas utilizadas é uma questão fundamental para impulsionar o uso de BIM integrado ao ACV e ao CCV. A interação entre as diferentes ferramentas e processos é necessária para o ganho de tempo e para o aproveitamento das análises de desempenho ambiental. O potencial do BIM no desenvolvimento de um projeto sustentável vai depender não somente das tecnologias aplicadas, mas também dessa integração entre elas.

\section{Referências}

[1] LOMBERA, J. S., APREA, I. G. A system approach to the environmental analysis of industrial buildings. Building and Environment. v. 45, p. 673-683, 2010. https://doiorg.ez10.periodicos.capes.gov.br/10.1016/j.buildenv.2009.08.012

[2] NAJJAR, M. FIGUEIREDO, K., HAMMAD, A. W. A. e HADDAD, A. Integrated optimization with building information modeling and life cycle assessment for generating energy efficient buildings. Applied Energy. v. 250 p. 1366-1382, 2019. https://doi.org/10.1016/j.apenergy.2019.05.101

[3] WONG J. K. W. e ZHOU J. Enhancing environmental sustainability over building life cycles through green BIM: A review. Autom Constr. V.57. p. 156-165. 2015. https://doiorg.ez10.periodicos.capes.gov.br/10.1016/j.autcon.2015.06.003

[4] KHASREEN M. M., BANFILL P. F. G, MENZIES GF. Life-cycle assessment and the environmental impact of buildings: a review. J Sustain. p. 674-701. 2009. https://doi.org/10.3390/su1030674

[5] RAMESH T, PRAKASH R, SHUKLA K. Life cycle energy analysis of buildings: An $\begin{array}{lllll}\text { overview. } & \text { Energy } & \text { Build. } & \text { V.42, }\end{array}$ https://doi.org/10.1016/j.enbuild.2010.05.007

[6] VERDAGUER B. S., LLATAS C, MARTINEZ A. G. Critical review of bim-based LCA. method to buildings. Energy Build. v.136 p. 110-120. 2017. https://doiorg.ez10.periodicos.capes.gov.br/10.1016/j.enbuild.2016.12.009

[7] SHERIF, K. Life Cycle Costing: Concept and Practice. OMEGA The Int. JI of Mgmt Sci. Vol. 9, No. 3. pp. 287 to 296. 1981. https://doi.org/10.1016/0305-0483(81)90035-9

[8] KIRK, S., J., e DELL'ISOLA, A. J. Life Cycle Costing for Design Professionals, McGrew-Hill Book Company, New York. 1995

[9] SPIEGELHALTER, T. ACHIEVING THE NET-ZERO-ENERGY-BUILDINGS “2020 AND 2030 TARGETS" WITH THE SUPPORT OF PARAMETRIC 3-D/4-D BIM DESIGN TOOLS. Journal of Green Building. V. 7, n. 2, p. 74-86, 2019. DOI: 10.3992/jgb.7.2.74

[10] RÖCK, M. ALEXANDER HOLLBERG, A. GUILLAUME HABERT, G. PASSER. A. LCA and BIM: Visualization of environmental potentials in building construction at early design stages. Building and Environment v.140 p.153-161. 2018. https://doi.org/10.1016/j.buildenv.2018.05.006

[11] MIHINDU, S. e ARA YICI, Y. Digital Construction through BIM Systems will drive the Re-engineering of Construction Business Practices. International Conference Visualisation p. 29-34, 2008 . DOI 10.1109 / VIS.2008.2 
[12] SZONYI, L. Building Information Modelling in the decision process of retrofitting the envelope of public buildings - a case study. Periodica polytechnica Civil Engineering. v. 54/2 p. 143-154, 2010. https://doi.org/10.3311/pp.ci.2010-2.10

[13] LEE, S., TAE, S., ROH. S. e KIM, T. Green Template for Life Cycle Assessment of Buildings Based on Building Information Modeling: Focus on Embodied Environmental Impact. Sustainability. v. 7, p. 16498-16512; 2015. doi:10.3390/su71215830

[14] SCHADE, J., OLOFSSON, T. e SCHREYER, M. Decision-making in a model-based design process, Construction Management and Economics, V. 29:4, p. 371-382, 2011. https://doi-org.ez10.periodicos.capes.gov.br/10.1080/01446193.2011.552510

[15] SANTOS, R.; COSTA, A. A; SILVESTRE, J. D. e PYL, L. Integration of LCA and LCC analysis within a BIM-based environment. Automation in Construction . v. 103 p. 127-149, 2019. https://doi.org/10.1016/j.autcon.2019.02.011

[16] OTI, A. H. e TIZANI, W. BIM extension for the sustainability appraisal of conceptual steel design. Advanced Engineering Informatics v. 29, p. 28-46, 2015. https://doi.org/10.1016/j.aei.2014.09.001

[17] WANG, E., SHEN, Z., e BARRYMAN, C. A building LCA case study using Autodesk Ecotect and BIM model. In T. Sulbaran, editors. 47th ASC Annual International Conference Proceedings. 2011. Disponível em: < https://digitalcommons.unl.edu/cgi/viewcontent.cgi?article=1005\&context=constructio nmgmt > Acesso em: 17 de dezembro de 2019.

[18] BASBAGILL, J.; FLAGER, F.; LEPECH, M. e FISCHER, M. Application of life-cycle assessment to early stage building design for reduced embodied environmental impacts, Build. Environ. v. 60, p. 81-92, 2013. https://doi.org/10.1016/j.buildenv.2012.11.009

[19] SHIN, Y.S.; CHO, K. BIM application to select appropriate design alternative with consideration of LCA and LCCA, Math. Probl. Eng. 2015. http://dx.doi.org/10.1155/2015/281640

[20] JRADE, A.; JALAEI, F. Integrating building information modelling with sustainability to design building projects at the conceptual stage, Build. Simul. v. 6 n. 4, p. 429-444, 2013. https://doi.org/10.1007/s12273-013-0120-0

[21] KEHILY, D.; UNDERWOOD, J. Embedding life cycle costing in 5D BIM, ITcon v. 22, p. 145-167, 2017. http://www.itcon.org/2017/8

[22] KORMAN, T. M. e KING, L. H. INDUSTRY Input for Construction Engineering and Management Courses: Development of a Building Systems Coordination Exercise for Construction Engineering and Management Students. Pract. Period. Struct. Des. Constr., v.19 n.1, p. 68-72, 2014. DOI: 10.1061/(ASCE)SC.1943-5576.0000189.

[23] VERDAGUER, B. S.; LLATAS, C.; MARTÍNEZ, A. G. e CÓZAR, J. C. G. BIM-Based LCA Method to Analyze Envelope Alternatives of Single-Family Houses: Case Study in Uruguay. J. Archit. Eng., v. 24, n. 3, 2018. DOI: 10.1061/(ASCE)AE.19435568.0000303.

[24] CAVAlliere,C., DEll'OSso, G. R., PIERUCCI, A. IANNONE, F. Life cycle assessment data structure for building information Modelling. Journal of Cleaner Production v.199, p.193-204, 2018. https://doi.org/10.1016/j.jclepro.2018.07.149 